\title{
The upgrade of the Pierre Auger Observatory with the Scintillator Surface Detector
}

\author{
Gabriella Cataldi $^{a, *}$ on behalf of the Pierre Auger ${ }^{b}$ Collaboration \\ (a complete list of authors can be found at the end of the proceedings) \\ ${ }^{a}$ INFN, Sezione di Lecce, via per Arnesano, 73100 Lecce, Italy \\ ${ }^{b}$ Observatorio Pierre Auger, Av. San Martín Norte 304, 5613 Malargüe, Argentina \\ E-mail: spokespersons@auger.org
}

Since its full commissioning in 2008, the Pierre Auger Observatory has consistently demonstrated its scientific productivity. A major upgrade of the Surface Detector array (SD) improves the capabilities of measuring the different components of extensive air showers. One of the elements of the upgrade consists of new Scintillator Surface Detectors (SSD) placed on top of the WaterCherenkov stations of the SD. At the Observatory, the integration of the SSD components and their deployment in the array is well advanced. In this paper, the main challenges and characteristics of the construction and installation will be reviewed. Started in 2016, an Engineering Array of twelve upgraded stations has been taking data in the field. In March 2019, a preproduction array of 77 SSDs started data acquisition with an adapted version of non-upgraded electronics. It is collecting events and proving the goodness of SSD design. Since December 2020, the upgraded electronics boards are being deployed in the field together with the photomultiplier tubes, increasing the number of SSD detectors, which are taking data continuosly with good stability. In this paper, the-long term performance of a subset of stations acquiring data for more than two years will be discussed. The data collected so far demonstrate the quality of the new detectors and the physics potential of the upgrade project.

$37^{\text {th }}$ International Cosmic Ray Conference (ICRC 2021)

July 12 th - 23rd, 2021

Online - Berlin, Germany

\footnotetext{
${ }^{*}$ Presenter
} 


\section{Introduction}

The upgrade [1, 2] of the Auger Observatory [3] follows from a decade of discovery and a recognition that shower-by-shower measurements of cosmic ray mass-related properties are essential to advance the field further [4]. The main scientific aspects motivating the upgrade are the lack of information on the primary mass composition at the highest energies, the observation that the averaged mass tends to increase at the highest energies complicating the anisotropy search, and the discrepancies among the hadronic interaction models predictions and the measured shower parameters. The upgrade consists of new plastic scintillator detectors (SSD) on top of the Water Cherenkov Detector (WCD) of the Surface Detector array (SD), an additional small photomultiplier (sPMT) installed in the WCD for the extension of the dynamic range, and new SD electronics provided by an Upgraded Unified Board (UUB) [5]. Additionally, an underground muon detector [6] is going to be deployed beside each WCD in the $750 \mathrm{~m}$ region of the Observatory to provide direct muon measurements. The upgrade will also be complemented by extending the measurements of the existing fluorescence detector [7] into periods of higher night-sky background to increase their duty cycle. Finally, based on the Auger Radio Engineering Array (AERA) experience and results, a radio detector is also included on top of every WCD to improve the measurement of the inclined showers since the SSDs are mostly sensitive to the vertical ones [8, 9].

In March 2019, the first 77 SSDs deployed had been equipped with photomultiplier tubes and put into data acquisition using an adapted version of the old electronics. In this paper, the data presented, unless explicitly stated, regard these 77 detectors. An overview of the performance of the UUB can be found in [5].

\section{The Scintillator Surface Detector}

The SSD, a thin scintillation detector, which is mounted above and triggered by the larger WCD below it, provides a robust and well-understood way of particle detection. Complementing the WCD of the surface array with scintillator detectors will provide a way to sample shower particles with detectors having very different responses to the muonic and electromagnetic components. An SSD consists of a box of $3.8 \mathrm{~m} \times 1.3 \mathrm{~m}$, containing two scintillator panels, each composed of extruded polystyrene scintillator bars of about $1.6 \mathrm{~m}$ length, $5 \mathrm{~cm}$ width, and $1 \mathrm{~cm}$ thickness. The two panels are enclosed in light-tight, weatherproof enclosures and are mounted on top of the existing WCD with a strong support frame, as shown in Fig.1(a). The scintillator light is read out with wavelength-shifting fibres inserted into straight extruded holes in the scintillator bars, which are bundled and optically coupled to a single photomultiplier tube (PMT), as shown in Fig.1(b). The fibers, Kuraray Y11(300)M S-type, are positioned following the grooves of the routers at both ends in a "U" configuration. They are bundled in a PMMA cylinder, whose front window is connected to a single PMT (Fig.1(b)). The PMT is a bi-alkali Hamamatsu R9420, 1.5" diameter, with $18 \%$ quantum efficiency at a wavelength of $500 \mathrm{~nm}$. For consistency with the associated WCD, the dynamic range in the SSD must span from the signal of a single particle, as needed for calibration, to large signals, up to $10^{4}$ Minimum Ionizing Particles (MIP). The anode current of the SSD PMT is split into two different channels, the first one amplified (High Gaing=HG) and the other attenuated (Low Gain=LG), to achieve the appropriate dynamic range. The type of PMT for the SSD has been 


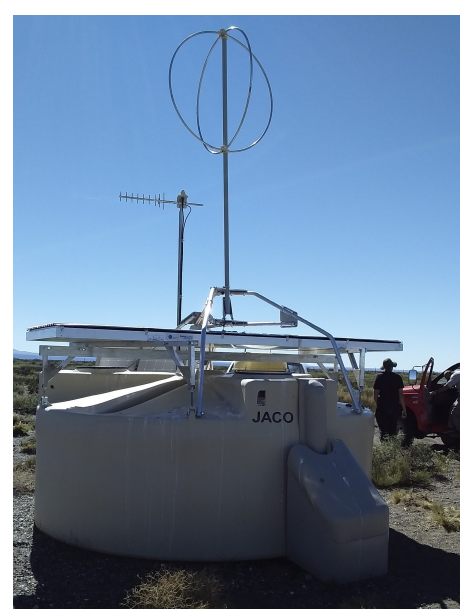

(a)

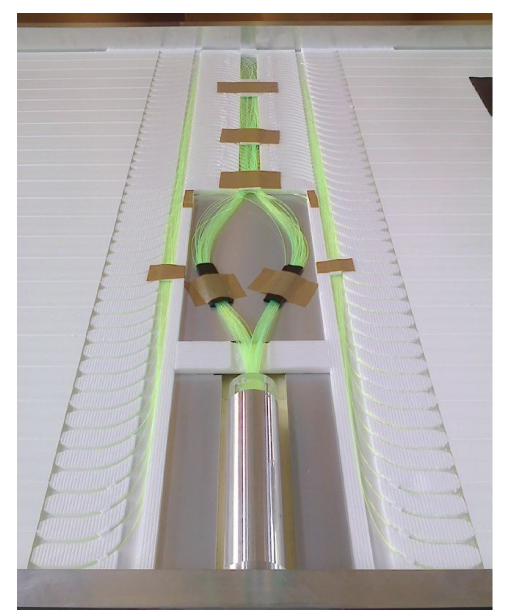

(b)

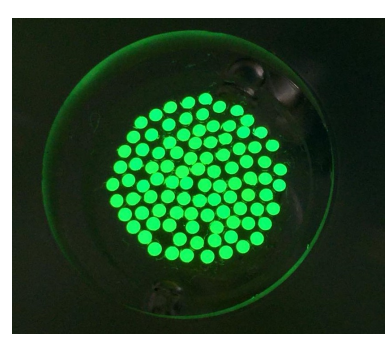

(c)

Figure 1: (a) One of the detectors of the SD array after the upgrade, featuring the SSD on top of the WCD; (b) Picture of the inner part of an SSD detector with green wavelength-shifting fibers routed from scintillators and guided in two bundles to the photomultiplier tube, housed in the aluminum support; (c) Picture of a PMMA front window of the cylinder where the wavelength-shifting fibres are bundled.

chosen also based on its excellent linear response when operated at LG, being linear within 5\% for peak currents up to $150 \mathrm{~mA}$ (for a gain at $7 \times 10^{5}$ ). The power supply of the PMT is based on a custom-made design manufactured by the ISEG company.

\section{Production and test of the detectors}

The assembly of the SSD was distributed among different European research institutes that are all members of the collaboration. Dedicated mechanical tools were developed and prepared prior to the assembly process, and a procedure has been agreed upon and written in detail to ensure a uniform quality among the different assembly sites. A detailed presentation of the procedures and tests of the detectors can be found in [10].

All the detectors have been individually tested with cosmic-ray muons to check the light tightness and to determine the detector response to a reference minimum-ionizing particle. For the test, every assembly site has organized a specific setup using the available detectors, the materials, and the areas of expertise of the research team. A cross-check of the performance among the sites has been carried on a subsample of detectors by testing few modules assembled in the different institutes and using one measurement setup as reference. The uniformity in the response of the SSD detectors can be measured via external trackers (e.g., planes of limited streamer tubes) on a muon tower setup. Fig.2(a) shows the average logarithm of the deposited charges of the particles depending on the position of intersection with the scintillator planes in $1 \mathrm{~cm} \times 1 \mathrm{~cm}$ bins for one of the SSD as measured in a muon tower.

The large-scale production of the 1518 detectors is now completed. All the tests have been evaluated, and the test results are being uploaded into a dedicated database that also contains the assembly and production details to ensure the traceability of procedures and materials used. To 


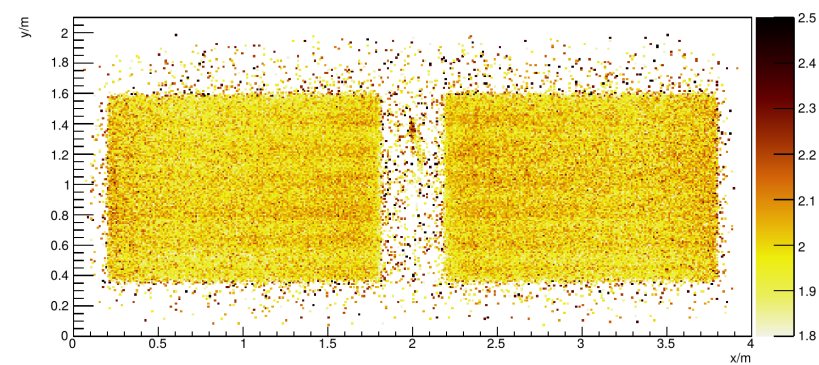

(a)

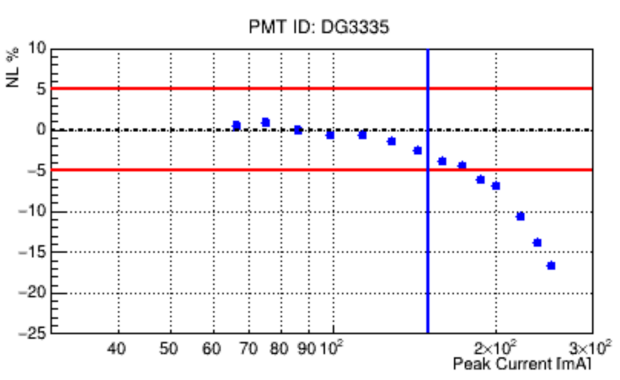

(b)

Figure 2: (a) The uniformity in the response of one of the SSD detectors as measured in a muon tracker; (b) Linearity curve for an SSD-PMT at a gain of $7 \times 10^{5}$.

achieve the needed performance, the single PMT used in each SSD requires excellent linearity. Two PMT test facilities $[11,12]$ were developed within the European research institutes of the collaboration to validate the features of each PMT, especially the linear response when operated at low gain. The testing sites have a specific setup and perform a cross-check on a subsample of detectors. Fig.2(b) shows the deviation from linearity as a function of the peak anode current for one of the PMTs at a reference gain of $7 \times 10^{5}$. All the PMTs (1590), once tested, are shipped to Argentina, where they are deployed in the experimental field together with the UUBs.

\section{Deployment in the array and monitoring}

The full production of SSDs has been shipped to Argentina. Their deployment in the experimental field, which started in October 2018, is now well advanced. The test of the PMTs is also complete, and the last batches will soon be shipped to Argentina. Prior to the deployment, the SSDs are prepared at the assembly building by the local Argentinean team. The preparation includes the placement of a box for the service of the electronics connection and the positioning of a corrugated roof on top of the detector. Moreover, the local team has the challenging task of transporting the SSDs to the different WCD stations, often located in hard-to-reach areas.

The online monitoring system [3], which overviews the operation and maintenance of the SD, includes the status of the deployment and the control of SSDs operation. In Fig.3(a) the map of the Pierre Auger Surface Detector array, including the SSD detectors, is shown. All colored points present one WCD running detector: the deployed SSDs that will be equipped with PMTs and new electronics, as soon as these components become available are in yellow, the SSDs connected to PMTs and taking data without the upgraded electronics that are the main interest of this paper are in orange, UUB and SSD taking data through the new electronics are marked in red, and finally the purple dots represent the WCDs not yet instrumented with SSDs. Not every WCD will be covered with SSD. As seen in Fig.3(a), apart from some specific small exceptions, an external ring of WCDs without SSD is present. Indeed, the SD stations located at the border of the array will not be equipped with SSDs. Around 1400 out of the total number of Auger surface detectors will be instrumented with SSD. The remaining detectors will be used either as spares or on specific parts of the experimental region (e.g., on doublets that are detectors located few meters apart or in the 


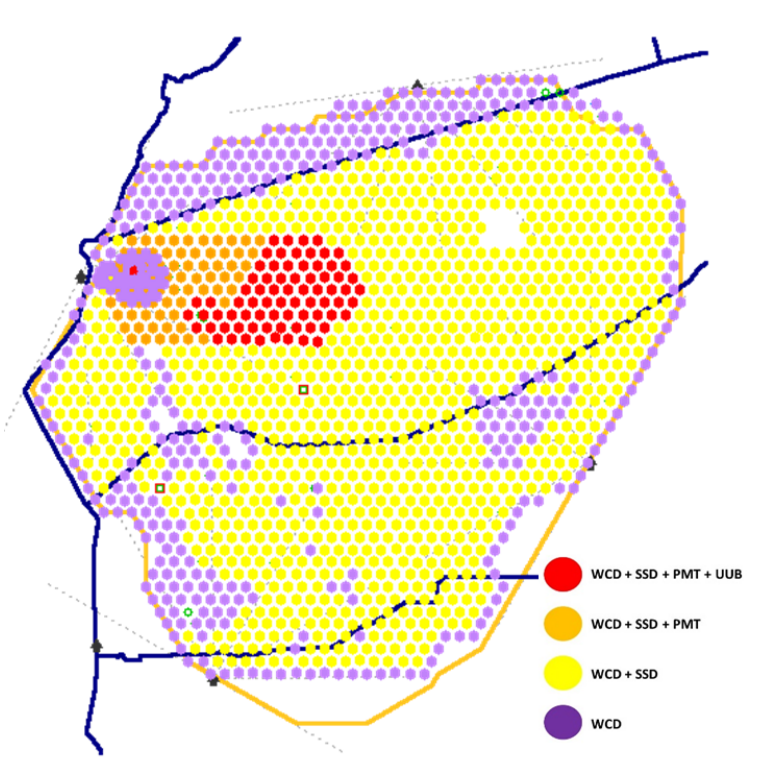

(a)

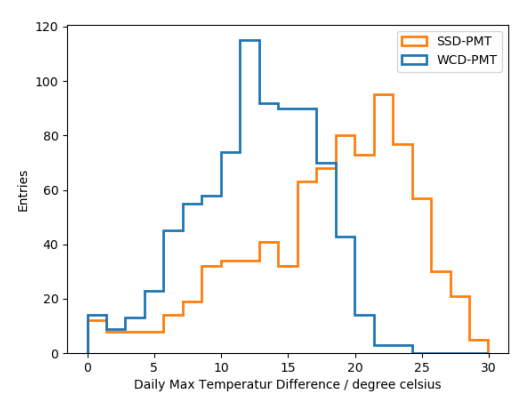

(b)

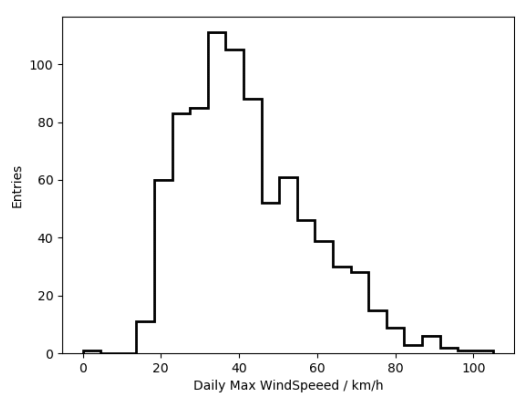

(c)

Figure 3: (a) Array status (at 10th June 2021) as seen from the online monitoring map of the Pierre Auger Surface Detector including the SSD detectors; (b) Diurnal temperature variation measured in the WCD and in the SSD for one of the detectors of the preproduction array; (c) Maximum wind speed monitored in the area of the preproduction array.

750m sub-array where the SDs are more densely spaced). The full array will contain the upgraded electronics and will be fully functional in trigger capabilities. A strict reconstruction condition requires that in a triggered event, the station with the highest signal is surrounded by six detectors in operation at the time of detection. With the exclusion of the external ring, the total array upgraded with SSDs will be about $90 \%$ of the full array of the observatory, but the global acceptance will be unchanged since the only showers involved will be those which have the core on or outside the external ring. These showers being anyway excluded at the reconstruction level. Moreover it should be noted that even in this border area, the reconstruction of events will be enriched by the data provided by the neighboring stations with SSDs.

The environment to which all SD detectors, including SSDs, are exposed is somewhat hostile. At $1400 \mathrm{~m}$ a.s.l. and with clear skies, day-night temperature variations often exceed $20^{\circ} \mathrm{C}$. The terrain properties vary over the $3000 \mathrm{~km}^{2}$ of the array, and some detectors have to face high salinity corrosive air, while others face dusty air from the sandy ground. The field area is subject to severe wind speeds, especially during strong thunderstorms. Therefore all the detector elements must be firmly anchored. Various sensors are installed to monitor the whole array accurately. The graph in Fig.3(b) shows the diurnal variation over the full data-taking period of two years for one of the SSD stations of the array. The temperature sensor is positioned inside the PMT housing. During the data acquisition period (see Fig 3(c)), strong wind conditions of more than $100 \mathrm{~km} / \mathrm{h}$ have been measured, and all the SSD detectors did withstand the elements. Given the environment, the extensions of the array, and the dimension of the detectors, the full deployment is definitively a 


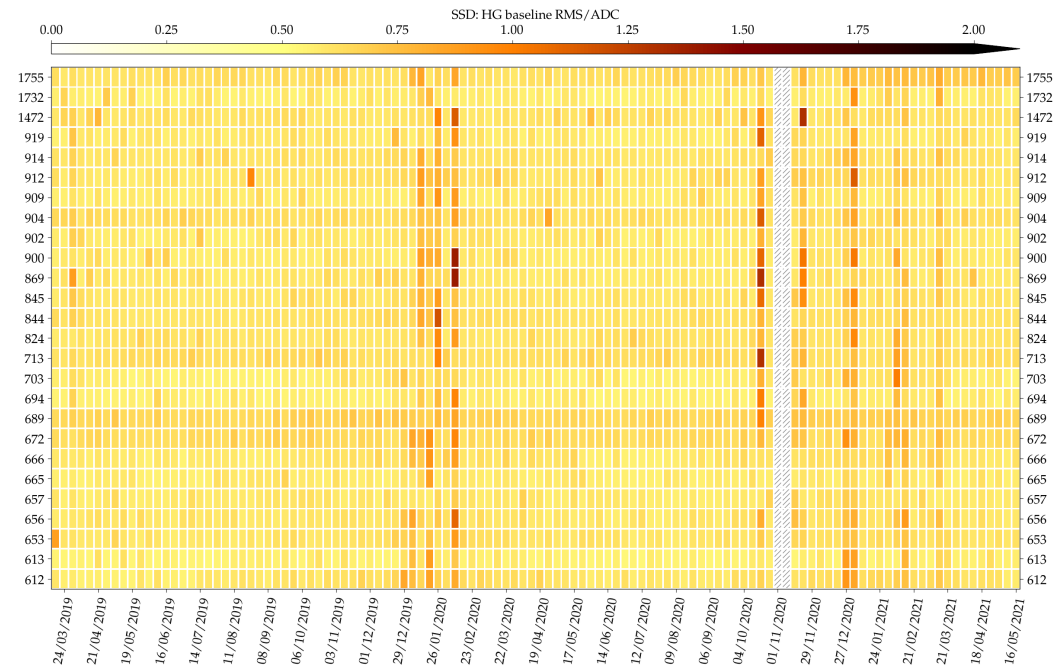

Figure 4: Evolution of the RMS in ADC counts for the PMTs trace (HG channel) for a subsample of SSD of the preproduction array.
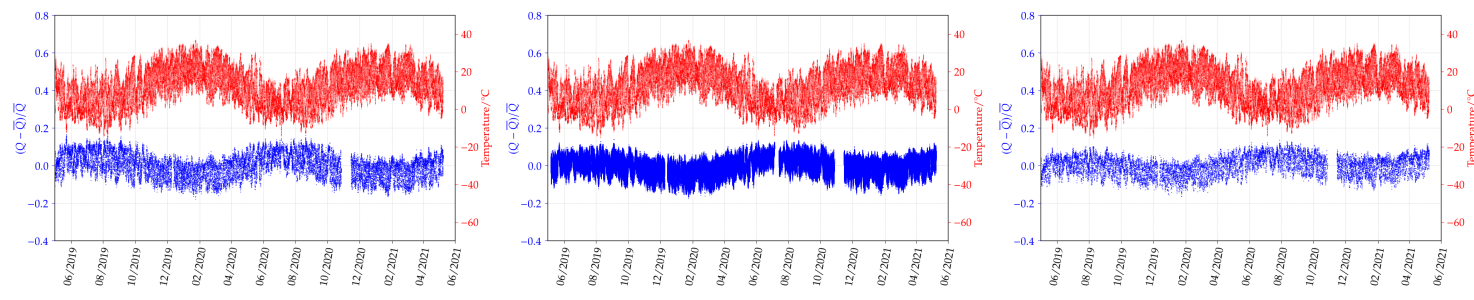

Figure 5: Evolution of the oscillation around an average MIP value (in blue) and evolution of the temperature (in red) for 3 SSDs of the array.

demanding task, especially complicated by the Covid-19 pandemic situation.

\section{Status of the array and stability of Operations}

Started in 2016, an Engineering Array of twelve upgraded SD stations has been operating in the field, with the aim of testing and monitoring the performance of the upgraded detectors. Additionally, in March 2019, a preproduction array of 77 SSDs has been deployed and put into operation with an adapted version of the previous electronics. This preproduction array is marked in orange in Fig.3(a)). Fig.4 shows the evolution along the data acquisition period of the weekly width average (RMS) of the HG baseline expressed in ADC counts for a subsample of detectors of the SSD preproduction array. On the vertical scale, the identification number of the WCDs are reported. The uniformity in horizontal rows clearly shows the stability of the detectors, while the vertical patterns evidence the presence of thunderstorms or communication problems in the array. Moreover, starting from December 2020, as soon as the UUBs are available, the fully upgraded SDs are put into acquisitions, increasing the fraction of the upgraded array. They are taking data continuosly with good stability.

The SSD calibration is based on the signal of a MIP going through the detector. The charge deposited by a MIP corresponds to a peak of the charge distribution, and the value of the MIP 


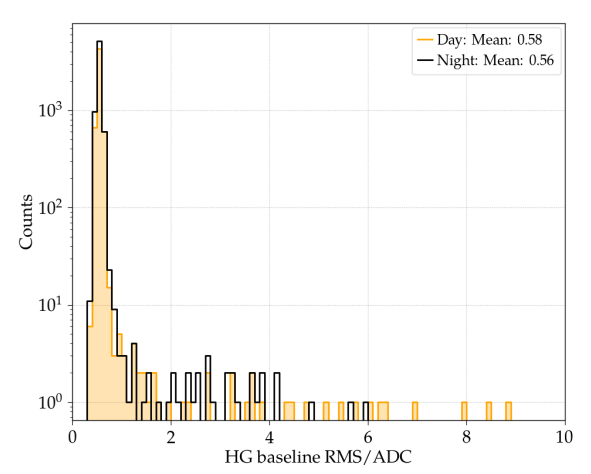

(a)

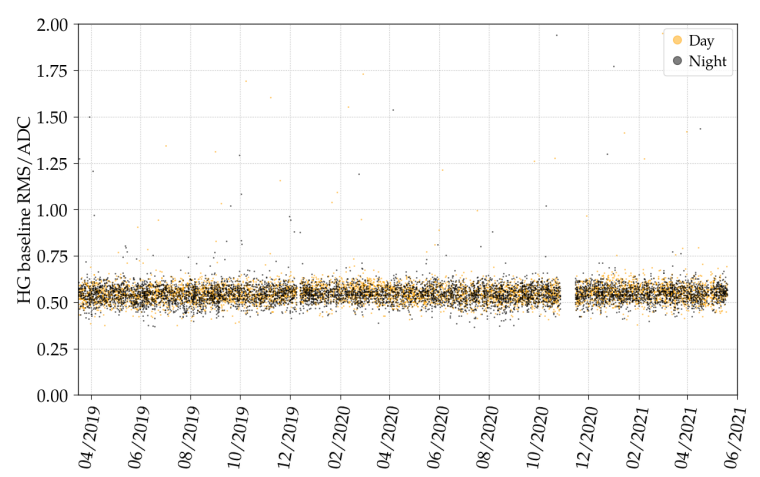

(b)

Figure 6: (a) Average Baseline width (RMS) and (b) its evolution with time for one of the SSD of the array.

average charge can be inferred from the fit to that peak. The values of the MIP charge (Q) vary from station to station due to differences in gain of the PMT, which might be affected by changes in the temperature or in the high voltage, for example. Therefore, studying the time evolution of the charge might give insights into the performance of the detectors.

For every SSD, it is possible to measure the charge corresponding to the MIP and to determine the oscillation around a mean value calculated as $(Q-\bar{Q}) / \bar{Q}$. Fig.5 shows the evolution of this variable with time (in blue), as well as the variation of temperature measured in the SSD PMT housing (in red) for three SSDs of the preproduction array during the full data taking period. The correlation with the temperature of a specific detector is clearly evident, showing evidence for diurnal and seasonal behaviours.

All SSD detectors have been tested for light-tightness at the production sites, however, they can be spoiled during shipment, deployment, or more generally because of unforeseen damages or aging. Several variables are measured and monitored with the aim to establish the parameters which have to be regularly monitored during data taking. A way to check the light-tightness of a detector is to compare the variation of the baseline during day-night. Fig.6 shows the RMS distribution of one SSD PMT baseline for day-night comparison of events for the full data acquisition period, together with its evolution over time. An analogy in response between the signals occurring at night-time and the ones at daytime, as the one shown in Fig.6, is evidence of "light-tight detector". Vice versa, the presence of a larger RMS when the detectors are exposed to the daylight indicates that the detector is suffering from a light leak. Other variables, as the asymmetry around the baseline, are investigated in combination with the RMS to discriminate the various noise patterns and sources.

The correlation between the signals reconstructed in the WCD and in the corresponding SSD is shown in Fig.7, where both scales are expressed in the corresponding physical units: for the WCD, Vertical Equivalent Muon (VEM), average charge deposited by a vertical and central through-going muon; and MIP for the SSD. This plot refers to fully upgraded stations, including UUB and SPMT. The signals in the WCD are measured up to saturation $(\approx 700$ VEM) by the large PMTs in the WCD tanks (blue dots). In the superposition region and above the saturation region, they are derived from the sPMT (red dots). The non-saturated range of the WCD extends to more than $\approx$ 20000 VEM with the sPMT and an independent measurement of the signal is provided by SSD. 


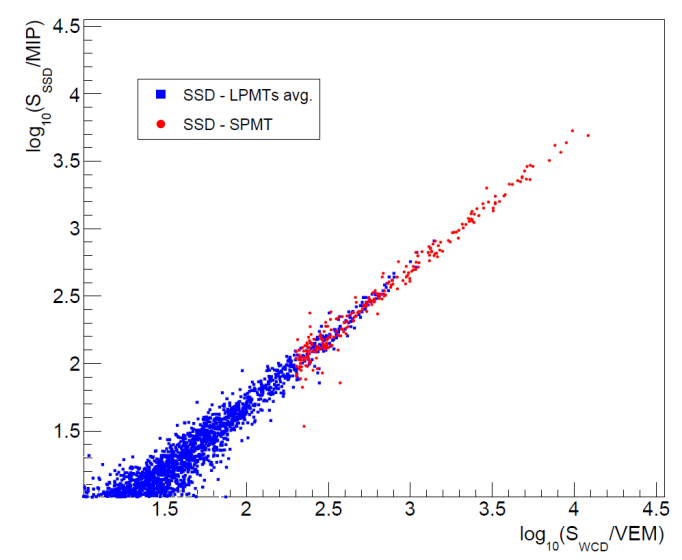

Figure 7: Correlation of signals in SSD versus WCD for the events reconstructed with the fully upgraded array. The large PMTs are used up to the saturation (blue dots); the measurements are extended further by means of the small PMT (red dots)

The correlation obtained demonstrates the validity of the calibration procedures and shows that the required dynamic range in the upgraded Surface Detector is covered up to the highest particle densities.

\section{Conclusions and Acknowledgements}

The production of the SSD detectors of the Pierre Auger Observatory, as well as their transportation to Argentina, has been completed. In spite of the Covid-19 pandemic, the deployment of SSDs in the full array will be completed before the end of 2021. The new electronics is in the production phase. The PMTs are gradually being installed together with the new electronics. All the SSDs deployed in the observatory surface array are foreseen to be in data acquisition at the end of 2022. The detectors are operating smoothly and with stability in agreement with the requirements.

The successful installation, commissioning, and operation of the Pierre Auger Observatory would not have been possible without the strong commitment and effort from the technical and administrative staff in Malargüe. To them go our special thank for their invaluable support.

\section{References}

[1] A. Aab et al. [Pierre Auger Coll.], arXiv [1604.03637][astro-ph.IM].

[2] A. Castellina [for the Pierre Auger coll.], EPJ WEB CONF 210 (2019) 06002.

[3] A. Aab et al. [Pierre Auger Coll.], Nucl. Instrum. Meth. A 798 (2015) 172-213, [1502 . 01323].

[4] R. Engel [for the Pierre Auger coll.], in these proceedings.

[5] G. Marsella [for the Pierre Auger coll.], in these proceedings.

[6] A. M. Botti [for the Pierre Auger coll.], in these proceedings.

[7] J. Abraham et al. [Pierre Auger Coll.], Nucl. Instrum. Meth. A 620 (2010) 227-251, [0907.4282].

[8] F. Schlüter [for the Pierre Auger coll.], in these proceedings.

[9] T. Fodran [for the Pierre Auger coll.], in these proceedings.

[10] J. Pȩkala [for the Pierre Auger coll.], Proc. 36th Int. Cosmic Ray Conf., Madison (WI), U.S.A. (2019), PoS(ICRC2019)380, [1909.09073].

[11] M. Buscemi et al., J INSTRUM 15 (2020) P07011.

[12] J. Rautenberg et al., in these proceedings. 


\section{The Pierre Auger Collaboration}

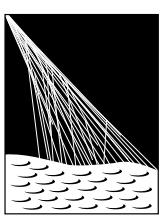

PIERRE

AUSGERVATORY

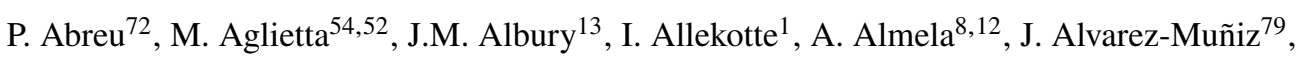
R. Alves Batista ${ }^{80}$, G.A. Anastasi ${ }^{63,52}$, L. Anchordoqui ${ }^{87}$, B. Andrada ${ }^{8}$, S. Andringa ${ }^{72}$, C. $\mathrm{Aramo}^{50}$, P.R. Araújo Ferreira ${ }^{42}$, J. C. Arteaga Velázquez ${ }^{67}$, H. Asorey $^{8}$, P. Assis ${ }^{72}$, G. Avila ${ }^{11}$, A.M. Badescu ${ }^{75}$, A. Bakalova ${ }^{32}$, A. Balaceanu ${ }^{73}$, F. Barbato ${ }^{45,46}$, R.J. Barreira Luz $^{72}$, K.H. Becker ${ }^{38}$, J.A. Bellido ${ }^{13,69}$, C. Berat ${ }^{36}$, M.E. Bertaina ${ }^{63,52}$, X. Bertou ${ }^{1}$, P.L. Biermann ${ }^{b}$, V. Binet ${ }^{6}$, K. Bismark ${ }^{39,8}$, T. Bister ${ }^{42}$, J. Biteau ${ }^{37}$, J. Blazek ${ }^{32}$, C. Bleve ${ }^{36}$, M. Boháčová ${ }^{32}$, D. Boncioli ${ }^{57,46}$, C. Bonifazi ${ }^{9,26}$, L. Bonneau Arbeletche ${ }^{21}$, N. Borodai ${ }^{70}$, A.M. Botti ${ }^{8}$, J. Brack ${ }^{d}$, T. Bretz ${ }^{42}$, P.G. Brichetto Orchera ${ }^{8}$, F.L. Briechle ${ }^{42}$, P. Buchholz ${ }^{44}$, A. Bueno ${ }^{78}$, S. Buitink ${ }^{15}$, M. Buscemi ${ }^{47}$, M. Büsken ${ }^{39,8}$, K.S. Caballero-Mora ${ }^{66}$, L. Caccianiga ${ }^{59,49}$, F. Canfora ${ }^{80,81}$, I. Caracas ${ }^{38}$, J.M. Carceller ${ }^{78}$, R. Caruso ${ }^{58,47}$, A. Castellina ${ }^{54,52}$, F. Catalanii ${ }^{19}$, G. Cataldi ${ }^{48}$, L. Cazon ${ }^{72}$, M. Cerda ${ }^{10}$, J.A. Chinellato ${ }^{22}$, J. Chudoba ${ }^{32}$, L. Chytka ${ }^{33}$, R.W. Clay ${ }^{13}$, A.C. Cobos Ceruttii ${ }^{7}$, R. Colalillo ${ }^{60,50}$, A. Coleman ${ }^{93}$, M.R. Coluccia ${ }^{48}$, R. Conceição ${ }^{72}$, A. Condorelli ${ }^{45,46}$, G. Consolati ${ }^{49,55}$, F. Contreras ${ }^{11}$, F. Convenga ${ }^{56,48}$, D. Correia dos Santos $^{28}$, C.E. Covault ${ }^{85}$, S. Dasso ${ }^{5,3}$, K. Daumiller ${ }^{41}$, B.R. Dawson ${ }^{13}$, J.A. Day ${ }^{13}$, R.M. de Almeida $^{28}$, J. de Jesús ${ }^{8,41}$, S.J. de Jong ${ }^{80,81}$, G. De Mauro ${ }^{80,81}$, J.R.T. de Mello Neto ${ }^{26,27}$, I. De Mitri ${ }^{45,46}$, J. de Oliveira ${ }^{18}$, D. de Oliveira Franco ${ }^{22}$, F. de Palma ${ }^{56,48}$, V. de Souza $^{20}$, E. De Vito ${ }^{56,48}$, M. del Río ${ }^{11}$, O. Deligny ${ }^{34}$, L. Deval ${ }^{41,8}$, A. di Matteo $^{52}$, C. Dobrigkeit ${ }^{22}$, J.C. D’Olivo ${ }^{68}$, L.M. Domingues Mendes ${ }^{72}$, R.C. dos Anjos ${ }^{25}$, D. dos Santos $^{28}$, M.T. Dova ${ }^{4}$, J. Ebr ${ }^{32}$, R. Engel ${ }^{39,41}$, I. Epicoco ${ }^{56,48}$, M. Erdmann ${ }^{42}$, C.O. Escobar ${ }^{a}$, A. Etchegoyen ${ }^{8,12}$, H. Falcke ${ }^{80,82,81}$, J. Farmer ${ }^{92}$, G. Farrar ${ }^{90}$, A.C. Fauth ${ }^{22}$, N. Fazzini ${ }^{a}$, F. Feldbusch ${ }^{40}$, F. Fenu ${ }^{54,52}$,

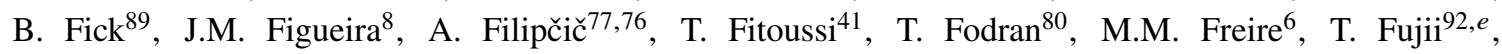
A. Fuster $^{8,12}$, C. Galea ${ }^{80}$, C. Galelli ${ }^{59,49}$, B. García ${ }^{7}$, A.L. Garcia Vegas ${ }^{42}$, H. Gemmeke ${ }^{40}$, F. Gesualdi ${ }^{8,41}$, A. Gherghel-Lascu ${ }^{73}$, P.L. Ghia ${ }^{34}$, U. Giaccari ${ }^{80}$, M. Giammarchi ${ }^{49}$, J. Glombitza ${ }^{42}$, F. Gobbi ${ }^{10}$, F. Gollan ${ }^{8}$, G. Golup ${ }^{1}$, M. Gómez Berisso ${ }^{1}$, P.F. Gómez Vitale ${ }^{11}$, J.P. Gongora ${ }^{11}$, J.M. González ${ }^{1}$, N. González ${ }^{14}$, I. Goos ${ }^{1,41}$, D. Góra ${ }^{70}$, A. Gorgi ${ }^{54,52}$, M. Gottowik ${ }^{38}$, T.D. Grubb ${ }^{13}$, F. Guarino ${ }^{60,50}$, G.P. Guedes ${ }^{23}$, E. Guido ${ }^{52,63}$, S. $\mathrm{Hahn}^{41,8}$, P. $\mathrm{Hamal}^{32}$, M.R. Hampel ${ }^{8}$, P. Hansen ${ }^{4}$, D. Harari ${ }^{1}$, V.M. Harvey ${ }^{13}$, A. Haungs ${ }^{41}$, T. Hebbeker ${ }^{42}$, D. Heck ${ }^{41}$, G.C. Hill ${ }^{13}$, C. Hojvat ${ }^{a}$, J.R. Hörandel ${ }^{80,81}$, P. Horvath ${ }^{33}$, M. Hrabovský ${ }^{33}$, T. Huege ${ }^{41,15}$, A. Insolia ${ }^{58,47}$, P.G. Isar $^{74}$, P. Janecek ${ }^{32}$, J.A. Johnsen ${ }^{86}$, J. Jurysek ${ }^{32}$, A. Kääpä ${ }^{38}$, K.H. Kampert ${ }^{38}$, N. Karastathis ${ }^{41}$, B. Keilhauer ${ }^{41}$, J. Kemp ${ }^{42}$, A. Khakurdikar ${ }^{80}$, V.V. Kizakke Covilakam $^{8,41}$, H.O. Klages ${ }^{41}$, M. Kleifges ${ }^{40}$, J. Kleinfeller ${ }^{10}$, M. Köpke ${ }^{39}$, N. Kunka ${ }^{40}$, B.L. Lago ${ }^{17}$, R.G. Lang ${ }^{20}$, N. Langner ${ }^{42}$, M.A. Leigui de Oliveira ${ }^{24}$, V. Lenok $^{41}$, A. Letessier-Selvon ${ }^{35}$, I. LhenryYvon $^{34}$, D. Lo Presti ${ }^{58,47}$, L. Lopes ${ }^{72}$, R. López ${ }^{64}$, L. Lu ${ }^{94}$, Q. Luce ${ }^{39}$, J.P. Lundquist ${ }^{76}$, A. Machado Payeras $^{22}$, G. Mancarella ${ }^{56,48}$, D. Mandat ${ }^{32}$, B.C. Manning ${ }^{13}$, J. Manshanden ${ }^{43}$, P. Mantsch ${ }^{a}$, S. Marafico ${ }^{34}$, A.G. Mariazzi ${ }^{4}$, I.C. Mariş ${ }^{14}$, G. Marsella ${ }^{61,47}$, D. Martello ${ }^{56,48}$, S. Martinelli ${ }^{41,8}$, O. Martínez Bravo ${ }^{64}$, M. Mastrodicasa ${ }^{57,46}$, H.J. Mathes ${ }^{41}$, J. Matthews ${ }^{88}$, G. Matthiae ${ }^{62,51}$, E. Mayotte ${ }^{38}$, P.O. Mazur ${ }^{a}$, G. MedinaTanco $^{68}$, D. Melo $^{8}$, A. Menshikov ${ }^{40}$, K.-D. Merenda ${ }^{86}$, S. Michal ${ }^{33}$, M.I. Micheletti ${ }^{6}$, L. Miramonti ${ }^{59,49}$, S. Mollerach ${ }^{1}$, F. Montanet ${ }^{36}$, C. Morello ${ }^{54,52}$, M. Mostafá ${ }^{91}$, A.L. Müller ${ }^{8}$, M.A. Muller ${ }^{22}$, K. Mulrey ${ }^{15}$, R. Mussa ${ }^{52}$, M. Muzio ${ }^{90}$, W.M. Namasaka ${ }^{38}$, A. Nasr-Esfahani ${ }^{38}$, L. Nellen ${ }^{68}$, M. Niculescu-Oglinzanu ${ }^{73}$, M. Niechciol ${ }^{44}$, D. Nitz ${ }^{89}$, D. Nosek ${ }^{31}$, V. Novotny ${ }^{31}$, L. Nožka ${ }^{33}$, A Nucita ${ }^{56,48}$, L.A. Núñez ${ }^{30}$, M. Palatka ${ }^{32}$, J. Pallotta ${ }^{2}$, P. Papenbreer ${ }^{38}$, G. Parente ${ }^{79}$, A. Parra ${ }^{64}$, J. Pawlowsky ${ }^{38}$, M. Pech ${ }^{32}$, F. Pedreira ${ }^{79}$, J. Pȩkala ${ }^{70}$, R. Pelayo ${ }^{65}$, J. Peña-Rodriguez ${ }^{30}$, E.E. Pereira Martins ${ }^{39,8}$, J. Perez Armand ${ }^{21}$, C. Pérez Bertolli $^{8,41}$, M. Perlin ${ }^{8,41}$, L. Perrone ${ }^{56,48}$, S. Petrera ${ }^{45,46}$, T. Pierog ${ }^{41}$, M. Pimenta ${ }^{72}$, V. Pirronello ${ }^{58,47}$, M. Platino ${ }^{8}$, B. Pont $^{80}$, M. Pothast ${ }^{81,80}$, P. Privitera ${ }^{92}$, M. Prouza ${ }^{32}$, A. Puyleart ${ }^{89}$, S. Querchfeld ${ }^{38}$, J. Rautenberg ${ }^{38}$, D. Ravignani ${ }^{8}$, M. Reininghaus ${ }^{41,8}$, J. Ridky ${ }^{32}$, F. Riehn ${ }^{72}$, M. Risse ${ }^{44}$, V. Rizi ${ }^{57,46}$, W. Rodrigues de Carvalho ${ }^{21}$, J. Rodriguez Rojo ${ }^{11}$, M.J. Roncoroni ${ }^{8}$, S. Rossoni ${ }^{43}$, M. Roth ${ }^{41}$, E. Roulet ${ }^{1}$, A.C. Rovero ${ }^{5}$, P. Ruehl ${ }^{44}$, A. Saftoiu ${ }^{73}$, F. Salamida ${ }^{57,46}$, H. Salazar ${ }^{64}$, G. Salina ${ }^{51}$, J.D. Sanabria Gomez ${ }^{30}$, F. Sánchez ${ }^{8}$, E.M. Santos ${ }^{21}$, E. Santos ${ }^{32}$, F. Sarazin ${ }^{86}$, R. Sarmento ${ }^{72}$, C. Sarmiento-Cano ${ }^{8}$, R. Sato ${ }^{11}$, 
P. Savina ${ }^{56,48,34,94}$, C.M. Schäfer ${ }^{41}$, V. Scherini ${ }^{56,48}$, H. Schieler ${ }^{41}$, M. Schimassek ${ }^{39,8}$, M. Schimp ${ }^{38}$, F. Schlüter ${ }^{41,8}$, D. Schmidt ${ }^{39}$, O. Scholten ${ }^{84,15}$, P. Schovánek ${ }^{32}$, F.G. Schröder ${ }^{93,41}$, S. Schröder ${ }^{38}$, J. Schulte ${ }^{42}$, S.J. Sciutto ${ }^{4}$, M. Scornavacche ${ }^{8,41}$, A. Segreto ${ }^{53,47}$, S. Sehgal ${ }^{38}$, R.C. Shellard ${ }^{16}$, G. Sigl ${ }^{43}$, G. Silli ${ }^{8,41}$, O. Sima ${ }^{73, f}$, R. Šmída ${ }^{92}$, P. Sommers ${ }^{91}$, J.F. Soriano ${ }^{87}$, J. Souchard ${ }^{36}$, R. Squartini ${ }^{10}$, M. Stadelmaier ${ }^{41,8}$, D. Stanca ${ }^{73}$, S. Stanič ${ }^{76}$, J. Stasielak ${ }^{70}$, P. Stassi ${ }^{36}$, A. Streich ${ }^{39,8}$, M. Suárez-Durán ${ }^{14}$, T. Sudholz ${ }^{13}$, T. Suomijärvi ${ }^{37}$, A.D. Supanitsky ${ }^{8}$, Z. Szadkowski ${ }^{71}$, A. Tapia ${ }^{29}$, C. Taricco $^{63,52}$, C. Timmermans ${ }^{81,80}$, O. Tkachenko ${ }^{41}$, P. Tobiska ${ }^{32}$, C.J. Todero Peixoto ${ }^{19}$, B. Tomé ${ }^{72}$, Z. Torrès ${ }^{36}$, A. Travaini ${ }^{10}$, P. Travnicek ${ }^{32}$, C. Trimarelli ${ }^{57,46}$, M. Tueros ${ }^{4}$, R. Ulrich ${ }^{41}$, M. Unger ${ }^{41}$, L. Vaclavek ${ }^{33}$, M. Vacula ${ }^{33}$, J.F. Valdés Galicia ${ }^{68}$, L. Valore ${ }^{60,50}$, E. Varela ${ }^{64}$, A. Vásquez-Ramírez ${ }^{30}$, D. Veberič ${ }^{41}$, C. Ventura ${ }^{27}$, I.D. Vergara Quispe ${ }^{4}$, V. Verzi ${ }^{51}$, J. Vicha ${ }^{32}$, J. Vink ${ }^{83}$, S. Vorobiov ${ }^{76}$, H. Wahlberg ${ }^{4}$, C. Watanabe ${ }^{26}$, A.A. Watson ${ }^{c}$, M. Weber ${ }^{40}$, A. Weindl ${ }^{41}$, L. Wiencke ${ }^{86}$, H. Wilczyński ${ }^{70}$, M. Wirtz ${ }^{42}$, D. Wittkowski ${ }^{38}$, B. Wundheiler ${ }^{8}$, A. Yushkov $^{32}$,

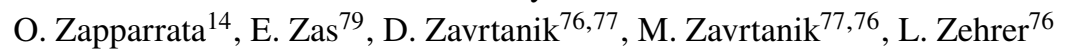

\footnotetext{
${ }^{1}$ Centro Atómico Bariloche and Instituto Balseiro (CNEA-UNCuyo-CONICET), San Carlos de Bariloche, Argentina

${ }^{2}$ Centro de Investigaciones en Láseres y Aplicaciones, CITEDEF and CONICET, Villa Martelli, Argentina

${ }^{3}$ Departamento de Física and Departamento de Ciencias de la Atmósfera y los Océanos, FCEyN, Universidad de Buenos Aires and CONICET, Buenos Aires, Argentina

${ }^{4}$ IFLP, Universidad Nacional de La Plata and CONICET, La Plata, Argentina

${ }^{5}$ Instituto de Astronomía y Física del Espacio (IAFE, CONICET-UBA), Buenos Aires, Argentina

${ }^{6}$ Instituto de Física de Rosario (IFIR) - CONICET/U.N.R. and Facultad de Ciencias Bioquímicas y Farmacéuticas U.N.R., Rosario, Argentina

${ }^{7}$ Instituto de Tecnologías en Detección y Astropartículas (CNEA, CONICET, UNSAM), and Universidad Tecnológica Nacional - Facultad Regional Mendoza (CONICET/CNEA), Mendoza, Argentina

${ }^{8}$ Instituto de Tecnologías en Detección y Astropartículas (CNEA, CONICET, UNSAM), Buenos Aires, Argentina

${ }^{9}$ International Center of Advanced Studies and Instituto de Ciencias Físicas, ECyT-UNSAM and CONICET, Campus Miguelete - San Martín, Buenos Aires, Argentina

${ }^{10}$ Observatorio Pierre Auger, Malargüe, Argentina

${ }^{11}$ Observatorio Pierre Auger and Comisión Nacional de Energía Atómica, Malargüe, Argentina

12 Universidad Tecnológica Nacional - Facultad Regional Buenos Aires, Buenos Aires, Argentina

${ }^{13}$ University of Adelaide, Adelaide, S.A., Australia

${ }^{14}$ Université Libre de Bruxelles (ULB), Brussels, Belgium

15 Vrije Universiteit Brussels, Brussels, Belgium

${ }^{16}$ Centro Brasileiro de Pesquisas Fisicas, Rio de Janeiro, RJ, Brazil

${ }^{17}$ Centro Federal de Educação Tecnológica Celso Suckow da Fonseca, Nova Friburgo, Brazil

${ }^{18}$ Instituto Federal de Educação, Ciência e Tecnologia do Rio de Janeiro (IFRJ), Brazil

${ }^{19}$ Universidade de São Paulo, Escola de Engenharia de Lorena, Lorena, SP, Brazil

${ }^{20}$ Universidade de São Paulo, Instituto de Física de São Carlos, São Carlos, SP, Brazil

${ }^{21}$ Universidade de São Paulo, Instituto de Física, São Paulo, SP, Brazil

22 Universidade Estadual de Campinas, IFGW, Campinas, SP, Brazil

${ }^{23}$ Universidade Estadual de Feira de Santana, Feira de Santana, Brazil

${ }^{24}$ Universidade Federal do ABC, Santo André, SP, Brazil

${ }^{25}$ Universidade Federal do Paraná, Setor Palotina, Palotina, Brazil

${ }^{26}$ Universidade Federal do Rio de Janeiro, Instituto de Física, Rio de Janeiro, RJ, Brazil

${ }^{27}$ Universidade Federal do Rio de Janeiro (UFRJ), Observatório do Valongo, Rio de Janeiro, RJ, Brazil

${ }^{28}$ Universidade Federal Fluminense, EEIMVR, Volta Redonda, RJ, Brazil

${ }^{29}$ Universidad de Medellín, Medellín, Colombia

${ }^{30}$ Universidad Industrial de Santander, Bucaramanga, Colombia

${ }^{31}$ Charles University, Faculty of Mathematics and Physics, Institute of Particle and Nuclear Physics, Prague, Czech Republic

32 Institute of Physics of the Czech Academy of Sciences, Prague, Czech Republic
} 
${ }^{33}$ Palacky University, RCPTM, Olomouc, Czech Republic

34 CNRS/IN2P3, IJCLab, Université Paris-Saclay, Orsay, France

${ }^{35}$ Laboratoire de Physique Nucléaire et de Hautes Energies (LPNHE), Sorbonne Université, Université de Paris, CNRSIN2P3, Paris, France

${ }^{36}$ Univ. Grenoble Alpes, CNRS, Grenoble Institute of Engineering Univ. Grenoble Alpes, LPSC-IN2P3, 38000 Grenoble, France

${ }^{37}$ Université Paris-Saclay, CNRS/IN2P3, IJCLab, Orsay, France

38 Bergische Universität Wuppertal, Department of Physics, Wuppertal, Germany

${ }^{39}$ Karlsruhe Institute of Technology (KIT), Institute for Experimental Particle Physics, Karlsruhe, Germany

${ }^{40}$ Karlsruhe Institute of Technology (KIT), Institut für Prozessdatenverarbeitung und Elektronik, Karlsruhe, Germany

${ }^{41}$ Karlsruhe Institute of Technology (KIT), Institute for Astroparticle Physics, Karlsruhe, Germany

${ }^{42}$ RWTH Aachen University, III. Physikalisches Institut A, Aachen, Germany

43 Universität Hamburg, II. Institut für Theoretische Physik, Hamburg, Germany

44 Universität Siegen, Department Physik - Experimentelle Teilchenphysik, Siegen, Germany

${ }^{45}$ Gran Sasso Science Institute, L'Aquila, Italy

46 INFN Laboratori Nazionali del Gran Sasso, Assergi (L'Aquila), Italy

${ }^{47}$ INFN, Sezione di Catania, Catania, Italy

${ }^{48}$ INFN, Sezione di Lecce, Lecce, Italy

${ }^{49}$ INFN, Sezione di Milano, Milano, Italy

${ }^{50}$ INFN, Sezione di Napoli, Napoli, Italy

${ }^{51}$ INFN, Sezione di Roma "Tor Vergata", Roma, Italy

52 INFN, Sezione di Torino, Torino, Italy

53 Istituto di Astrofisica Spaziale e Fisica Cosmica di Palermo (INAF), Palermo, Italy

54 Osservatorio Astrofisico di Torino (INAF), Torino, Italy

55 Politecnico di Milano, Dipartimento di Scienze e Tecnologie Aerospaziali , Milano, Italy

56 Università del Salento, Dipartimento di Matematica e Fisica "E. De Giorgi”, Lecce, Italy

57 Università dell'Aquila, Dipartimento di Scienze Fisiche e Chimiche, L'Aquila, Italy

58 Università di Catania, Dipartimento di Fisica e Astronomia, Catania, Italy

${ }^{59}$ Università di Milano, Dipartimento di Fisica, Milano, Italy

${ }^{60}$ Università di Napoli "Federico II", Dipartimento di Fisica "Ettore Pancini”, Napoli, Italy

${ }^{61}$ Università di Palermo, Dipartimento di Fisica e Chimica "E. Segrè", Palermo, Italy

62 Università di Roma "Tor Vergata", Dipartimento di Fisica, Roma, Italy

${ }^{63}$ Università Torino, Dipartimento di Fisica, Torino, Italy

${ }^{64}$ Benemérita Universidad Autónoma de Puebla, Puebla, México

${ }^{65}$ Unidad Profesional Interdisciplinaria en Ingeniería y Tecnologías Avanzadas del Instituto Politécnico Nacional (UPIITA-IPN), México, D.F., México

66 Universidad Autónoma de Chiapas, Tuxtla Gutiérrez, Chiapas, México

${ }^{67}$ Universidad Michoacana de San Nicolás de Hidalgo, Morelia, Michoacán, México

${ }^{68}$ Universidad Nacional Autónoma de México, México, D.F., México

${ }^{69}$ Universidad Nacional de San Agustin de Arequipa, Facultad de Ciencias Naturales y Formales, Arequipa, Peru

${ }^{70}$ Institute of Nuclear Physics PAN, Krakow, Poland

${ }^{71}$ University of Łódź, Faculty of High-Energy Astrophysics,Lódź, Poland

${ }^{72}$ Laboratório de Instrumentação e Física Experimental de Partículas - LIP and Instituto Superior Técnico - IST, Universidade de Lisboa - UL, Lisboa, Portugal

73 "Horia Hulubei” National Institute for Physics and Nuclear Engineering, Bucharest-Magurele, Romania

${ }^{74}$ Institute of Space Science, Bucharest-Magurele, Romania

75 University Politehnica of Bucharest, Bucharest, Romania

76 Center for Astrophysics and Cosmology (CAC), University of Nova Gorica, Nova Gorica, Slovenia

${ }^{77}$ Experimental Particle Physics Department, J. Stefan Institute, Ljubljana, Slovenia

78 Universidad de Granada and C.A.F.P.E., Granada, Spain

${ }^{79}$ Instituto Galego de Física de Altas Enerxías (IGFAE), Universidade de Santiago de Compostela, Santiago de Compostela, Spain 
${ }^{80}$ IMAPP, Radboud University Nijmegen, Nijmegen, The Netherlands

${ }^{81}$ Nationaal Instituut voor Kernfysica en Hoge Energie Fysica (NIKHEF), Science Park, Amsterdam, The Netherlands

82 Stichting Astronomisch Onderzoek in Nederland (ASTRON), Dwingeloo, The Netherlands

${ }^{83}$ Universiteit van Amsterdam, Faculty of Science, Amsterdam, The Netherlands

${ }^{84}$ University of Groningen, Kapteyn Astronomical Institute, Groningen, The Netherlands

85 Case Western Reserve University, Cleveland, OH, USA

86 Colorado School of Mines, Golden, CO, USA

${ }^{87}$ Department of Physics and Astronomy, Lehman College, City University of New York, Bronx, NY, USA

${ }^{88}$ Louisiana State University, Baton Rouge, LA, USA

${ }^{89}$ Michigan Technological University, Houghton, MI, USA

${ }^{90}$ New York University, New York, NY, USA

${ }^{91}$ Pennsylvania State University, University Park, PA, USA

92 University of Chicago, Enrico Fermi Institute, Chicago, IL, USA

93 University of Delaware, Department of Physics and Astronomy, Bartol Research Institute, Newark, DE, USA

94 University of Wisconsin-Madison, Department of Physics and WIPAC, Madison, WI, USA

${ }^{a}$ Fermi National Accelerator Laboratory, Fermilab, Batavia, IL, USA

${ }^{b}$ Max-Planck-Institut für Radioastronomie, Bonn, Germany

${ }^{c}$ School of Physics and Astronomy, University of Leeds, Leeds, United Kingdom

${ }^{d}$ Colorado State University, Fort Collins, CO, USA

${ }^{e}$ now at Hakubi Center for Advanced Research and Graduate School of Science, Kyoto University, Kyoto, Japan

$f$ also at University of Bucharest, Physics Department, Bucharest, Romania 